\title{
How Analogies Fit in a Framework for Supporting the Entrepreneurial Mind- set in an Electric Circuits Course
}

\section{Dr. Heath Joseph LeBlanc, Ohio Northern University}

Heath J. LeBlanc is an Associate Professor in the Electrical \& Computer Engineering and Computer Science Department at Ohio Northern University. He received his MS and PhD degrees in Electrical Engineering from Vanderbilt University in 2010 and 2012, respectively, and graduated summa cum laude with his BS in Electrical Engineering from Louisiana State University in 2007. His research interests include cooperative control of networked multi-agent systems, resilient and fault-tolerant control, and networked control systems. His teaching interests include controls and automation, electric circuits, signals and systems, engineering economics, electromagnetics, and integrating the entrepreneurial mindset with an engineering mindset in core engineering courses. He received the Professor Henry Horldt Outstanding Teaching Award in 2015.

\section{Dr. Khalid S. Al-Olimat P.E., Ohio Northern University}

Dr. Khalid S. Al-Olimat is professor and chair of the Electrical \& Computer Engineering and Computer Science Department at Ohio Northern University (ONU). He obtained his BS in Electrical Engineering from Far Eastern University in 1990, the MS in Manufacturing Engineering from Bradley University in 1994 and his PhD in Electrical Engineering from the University of Toledo in 1999. Dr. Al-Olimat joined ONU in fall of 1999. He teaches Circuits, electromagnetics, and the power engineering course sequence. Dr. Al-Olimat is the recipient of Henry Horldt Outstanding Teacher Award two times in 2004 and 2014, Herbert F. Alter Chair of Engineering in 2005, and the University of Toledo Outstanding Alumni Award in 2018. Dr. Al-Olimat has many publications in the areas of adaptive control, fuzzy control and machine drives in addition to engineering education. His areas of interest are power engineering, adaptive, fuzzy and intelligent control. Dr. Al-Olimat is a registered professional engineer in the State of Michigan and an ABET Program Evaluator.

Dr. Al-Olimat is a member and president of Phi Beta Delta, Honor Society for International Scholars (ONU), and a member of Tau Beta Pi Engineering Honor Society. He is also a senior member of IEEE. He served IEEE as Treasurer, Secretary, Vic-Chair, and Chair of IEEE-Lima section. He is serving the profession as a technical reviewer for many IEEE transaction journals in the areas of power and energy systems.

Dr. Al-Olimat is listed in Marquis Who's Who in Science and Engineering, Marquis Who's Who in American Education, Marquis Who's Who in America, Marquis Who's Who in the World and also Listed in Strathmore's Who's Who.

\section{Dr. Muhammad Ajmal Khan, Ohio Northern University}

Dr. M. Ajmal Khan is an Assistant Professor (tenure-track) in the Department of Electrical \& Computer Engineering and Computer Science (ECCS) at Ohio Northern University (ONU). He did his Ph.D. from The University of Western Ontario (UWO), London, Canada in wireless communications and data networks in 2016. His current research interests include wireless communications and networks, wireless systems security, and engineering education. He has actively participated in KEEN Innovating Curriculum with Entrepreneurial Mindset (ICE) Workshop in 2017 and KEEN National Conference 2018. He has actively incorporated various pedagogical techniques for Entrepreneurial Minded Learning (EML) in several courses including networks and data communications, electric circuits, systems design and communication systems.

\section{Dr. Firas Hassan, Ohio Northern University}

Firas Hassan is an associate professor at Ohio Northern University. He got his Ph.D. from The university of Akron. His research interest are in the area of embedded computing of real-time image processing techniques. 


\section{How Analogies Fit in a Framework for Supporting the Entrepreneurial Mindset in an Electric Circuits Course}

\section{Introduction}

In recent years, many engineering programs have placed an emphasis on instilling the entrepreneurial mindset (EM) in their graduates. The expected benefits of blending entrepreneurial skills and tendencies with engineering are great. It has even been postulated that engineers with an entrepreneurial inclination are the core drivers of economic growth in nations [1]. Many programs, both publicly and privately funded, have focused on entrepreneurship or the entrepreneurial mindset (EM) in engineering education. The National Science Foundation's ICorps program is one example on the public side, and the Kern Family Foundation's KEEN program (Kern Entrepreneurial Engineering Network) is an example on the private side.

KEEN is a growing network of over 35 U.S.-based academic institutions with the shared mission of integrating the EM in their undergraduate engineering programs [2]. The focus of KEEN is the EM rather than entrepreneurship. Whereas entrepreneurship focuses on entrepreneurial behaviors and actions, such as the creation of business ventures that deliver new products and services to the marketplace, the EM is a set of attitudes and dispositions that are the precursors to entrepreneurial action [3].

The KEEN framework for the EM is comprised of three major components: curiosity, connections, and creating value, the so-called 3Cs of the EM. Engineers with an entrepreneurial mindset should be curious about the world around them, to make the connections that enable insights, and ultimately identify opportunities to create value for themselves, their organizations, or, more broadly, society. In fact, it has been shown that curiosity has a positive and significant relationship with workplace learning and job performance [4]. Entrepreneurially minded engineers are not just the entrepreneurs with engineering degrees; they are able to fill both the roles of traditional staff engineers as well as leadership roles within organizations [5].

The set of teaching and learning strategies that aim to support the KEEN 3Cs framework for EM is referred to as Entrepreneurially Minded Learning (EML) [6]. Oftentimes, EML builds on active pedagogies, such as Project-Based Learning (PBL), and focuses holistically on opportunity recognition, stakeholder awareness, discovery, and value creation [7]. There are three entrepreneurial learning domains EML targets: affective factors (such as self-efficacy, intention, and motivation), thinking patterns (such as heuristics, logic types, mental shortcuts), and content knowledge and skills (business models, finance, market analysis, presentations) [6]. EML assignments or class activities may support these learning domains holistically or specifically target one or two of the learning domains (affective factors, thinking patterns, or knowledge and skills) or components of the EM (i.e., curiosity, connections, or creating value).

Many well established techniques in teaching and learning can support specific elements of the EM. For example, inquiry-based learning is positively linked with curiosity [8]. Metaphors and analogies can help students make connections between domains and improve understanding of 
abstract concepts [9]. From a constructivist viewpoint of learning, analogies are very effective for conceptual change [10]. Design thinking supports value creation [11]-[12].

Analogies, in particular, are very important for teaching abstract concepts, such as electric circuit concepts [13]. In [14], it is shown that analogies of electric current can aid student learning. Analogies consist of a target, which is the new idea to be learned, the source (or analog), which is the familiar domain, and a set of explicit comparisons between the structures of the source and target [9]. Analogies in which the relational structure between target and domain are highly developed, including higher-order similarities beyond surface features, better support inferences to be made using the analogy [9]. Elaborate analogies consisting of a systematic mapping between source and domain and accompanied by strong supporting imagery can further enhance the positive effects of analogy use [15]. A major concern when using analogies, is that unintended features of the source or even misconceptions of the source are mapped by the student to the target [16]. To avoid this unintended consequence of analogy use it is important to explicitly describe the features of the source that are to be mapped and explicitly discuss the limitations of the analogy, i.e. how the analogy is imprecise, as is suggested in the Teachingwith-Analogies Model [17]. Using multiple analogies for the same target is another strategy that has been demonstrated to avoid misconceptions being mapped to the target [18].

A major premise of this paper is that using circuit analogies to aid in teaching many of the fundamental concepts in an electric circuits course (e.g., Kirchoff's Laws, voltage division, loop analysis, etc.) better engages students, motivates them to learn the material, and helps them to connect the electric circuit topics to the source domain. It is well established in the literature that using analogies, especially two or more analogies for a specific target concept, can help students positively transfer their knowledge to new situations [19, p.5]. It is also well established that although analogies with deep relational structure are better for supporting inferences using analogical reasoning [9], imprecise analogies can also benefit student learning and avoid mapping unintended misconceptions if the misleading or imprecise aspects of the analogy are pointed out to the student [19, p.8]. One of the main contributions of this paper is a description of a set of novel circuit analogies that may be used in conjunction with other, more established and well-known circuit analogies, in order to provide the benefits of using multiples analogies [18].

A second premise of this paper is that exposing students to the thinking patterns associated with analogies and requiring students to articulate the deep structure between target and source domains support the thinking patterns used in making connections between disparate ideas that can lead to insights. Indeed, it is reported that the great Johannes Kepler developed many of his insights about gravity and planetary orbits using analogical reasoning [20]. Integrating information from many sources to gain insight is one of the example behaviors for the Connections component of the KEEN framework for the EM [2]. Hence, the use of analogies to promote student learning inside and outside of the classroom supports the Connections component of the KEEN framework for the EM, as it supports the thinking patterns that aid in making the connections to gain insight.

The contributions of this paper include a detailed list of novel circuit analogies, a framework for integrating EML in an electric circuits course, and emphasis of how analogies fit within that framework. The framework includes specific classroom activities and assignments to support 
curiosity, connections, and creating value. The analogies used in the electric circuits course are popular with students, as they help students make connections between circuit topics and other more familiar concepts and demystify the topics of the course.

The rest of the paper is organized as follows. Section 2 outlines the electric circuits course and describes the assignments and classroom activities that support curiosity, connections, and creating value in the course. Section 3 provides a list of novel circuit analogies used in the course. Finally, Section 4 provides student feedback from course reviews on the analogies used in the course and concludes the paper.

\section{Framework for Supporting the EM within an Electric Circuits Course}

The Electric Circuit course at the authors' institution is a sophomore-level, 4-credit hour required course and includes several engineering disciplines including electrical engineering, mechanical engineering, computer engineering, and engineering education. There are 3 lectures and a 3 hour lab each week. The topics of the course include: basic electrical quantities, circuit laws and theorems, analysis techniques, operational amplifiers, storage elements and first-order circuits, AC power analysis, three-phase systems, and ideal transformers. The course includes daily homework assignments, weekly quizzes and laboratory assignments, two midterm exams, and a comprehensive final exam. Due to the different majors present in the class, it is challenging to make the course attractive to all majors while covering the topics at a depth needed for electrical and computer engineering majors. To overcome this challenge, we have incorporated EML activities and assignments as part of the course. In what follows we categorize each of the EML assignments to one of the $3 \mathrm{Cs}$ in the KEEN framework; however, some learning objectives would more appropriately fall in the category of a different $\mathrm{C}$. We have categorized the assignments based on which of the $3 \mathrm{Cs}$ is most heavily emphasized.

\section{Curiosity}

The curiosity-related assignments aim to address the following learning objectives:

1) Develop a propensity to ask more questions.

2) Formulate salient questions related to electric circuit analysis and design.

3) Recognize and explore circuit knowledge gaps.

4) Identify and evaluate sources of information.

The assignments involve students generating their own questions suitable for exploration and following through with exploring one of those questions in a short exploratory research paper. There are a total of three question-submission assignments submitted throughout the semester. Each student is required to submit three questions in each question set assignment. Questions must be relevant to the material covered previously in the course. For Question Set 1, exploratory questions should be limited to the topics covered in the first 6 lectures, including electric circuit elements, Ohm's law, Kirchhoff's laws, serial and parallel connections, and voltage and current division. Question Set 2 includes the material on nodal analysis, loop analysis and circuit theorems. The material for Question Set 3 includes op-amp, capacitor and inductor, first order circuits, and AC fundamentals. 
As a classroom activity to support the question submission assignments, a question for each lecture is generated by the instructor to demonstrate as an example of a good question to be explored for the topic of the day. A single point rubric has been developed based on the template described in [21] and shared with the students (shown in Table 1). The instructor describes the elements of the rubric and scores the instructor-generated question using the rubric so students better understand both the elements of the rubric and the characteristics that make a good question. From time to time, a mediocre question has been generated intentionally to provide contrasting cases for students to see what makes a question bad. The first two to five minutes of each class are devoted to presenting the question of the day and discussing its rubric scoring. This classroom activity along with the three question submission assignments aim to support the first two learning objectives listed above. Students are exposed to many examples of salient questions related to electric circuit analysis and design and then are asked to produce their own questions.

Table 1: Single-point rubric for grading questions designed for exploring a topic

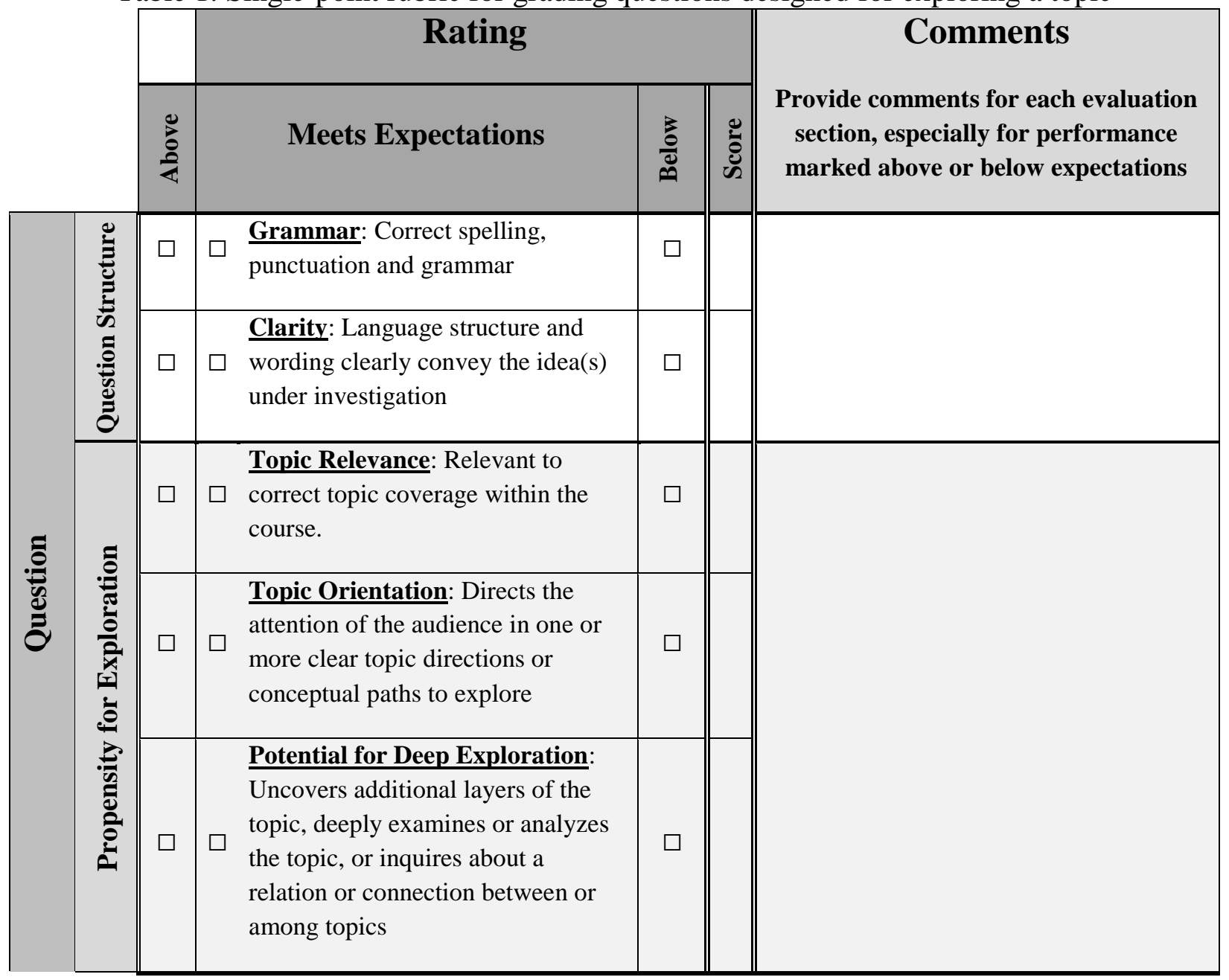

After the three question submission sets, a brief research paper is assigned that requires students to formulate an exploratory question, identify at least one credible and relevant source to use to 
explore the topic of the question, identify new questions that arise during the research process, and report their findings. It is important for students to demonstrate they are aware of what they do not know by formulating follow-up questions during the research. Doing so demonstrates an ability for students to engage in effective self-study, which supports life-long learning. Students complete the short report with an assessment of their sources found during the research process. This research paper support learning objectives (3) and (4) listed above.

\section{Creating Value}

To support creating value, a design project has been included in the course, which has elements of opportunity recognition, identification of suppliers and distributors, and articulation of a value proposition. The learning objectives associated with the design project are

1) Explores multiple solution paths in the circuit design process.

2) Identifies a market, market opportunities, or customer needs.

3) Modifies a design based on instructor feedback.

4) Gathers experimental and simulation data to support or refute circuit design ideas.

5) Craft a compelling value proposition tailored to specific stakeholders.

The temperature sensor design project described in [22] has been adapted for this course to include a problem framing and needs finding deliverable. Students are required to provide a problem statement for the need including a detailed description of the underlying need the temperature sensing circuit addresses and a list of the constraints and design criteria. Market data and analysis must be provided to justify the need as viable. This part of the assignment supports learning objective (2).

Students are required to individually develop alternative design solutions, analyze them, create a circuit simulation to validate the design, and submit the results. The individual nature of this deliverable reduces the likelihood of freeloading. Formative feedback is provided to students based on the initial designs and problem framing and needs finding deliverable. Students choose a design among the design alternatives to use for the remainder of the project, including prototype implementation and testing. This part of the assignment supports learning objectives (1) and (3).

The next stage of the project requires students to create detailed testing and implementation procedures for their chosen design. One lab period is dedicated for collecting the empirical data by carrying out the testing and implementation procedures developed by the students. This part of the assignment supports learning objective (4).

The final deliverable of the project is a written proposal that describes the identified opportunity, the circuit design, its analysis and simulation, a cost analysis based on a single bill of materials using two different suppliers (and selecting the best supplier based on the price), identification of a distributor, testing and implementation of the prototype, and the value proposition for the resulting product. This deliverable supports all 5 of the learning objectives. 


\section{Connections}

Throughout the course, many analogies are shared with students to help connect electric circuit concepts to real life experiences as well as other more familiar topics. The only learning objective associated with the analogy assignment is

1) Connect life experiences with electric circuit content.

By the end of the semester, students are required to submit a $1 / 2$ to 1 -page analogy reflection. In the assignment, students must either reflect on one of the analogies given throughout the course to connect it to a personal life experience, or to create their own analogy that connects the circuit content to another topic, and reflect on the connection to a life experience. The analogy should be stated, and the underlying deep structure between the source and target should be described.

For grading, the correctness of the statements made about the analogy and the related circuit topic are checked (i.e., the stated deep structure is sensible and correct). Also, how well the analogy is related to the student's own life experience is assessed. The grading of the analogy reflection assignment consists of the following breakdown, for a total of 50 points:

- Typesetting, format, and writing quality (paragraph structure, grammar, etc.) - 10 points

- Analogy statement - 10 points (either your own or transcribed from the notes)

- Analogy deep structure description - 20 points

- Connection to life experience - 10 points

Based on the experience of the instructors, students often struggle with the abstract concepts covered in the course when analogies are not given. To overcome this issue, we have incorporated well known analogies and created several novel analogies over time to help the students better learn the material and make the connection between the course material and their personal experience. Although this paper does not involve a research study, researchers have reported empirical benefits in applying analogies [19, Chapter 1].

\section{Circuit Analogies}

An analogy simply relates something new to something familiar. Analogies provide an effective strategy for teaching and learning and are typically used either to make abstract information more concrete or to organize complex information. Their purpose is to help students learn by comparing and connecting a new concept with something familiar. Some of the benefits of an effective analogy include motivating students, helping students to monitor and revise their thinking, remembering the content, constructing their own knowledge, overcoming misconceptions, and visualizing abstract concepts.

An effective analogy consists of four parts:

1. The target, or subject: the information to be learned.

2. The source, or analog: the familiar thing to which the new information is compared.

3. The connector: the means by which the subject and analog are compared.

4. The ground: the description of the similarities and differences between the subject and analog. 
To facilitate the use of analogies, the ABCDE method of constructing an analogy is considered [23].
A. Analyze the subject: what is it you most want the learners to understand about the subject?
B. Brainstorm potential analogs: what concrete items share the important features you have identified?
C. Choose the analog: which candidate analog has the best combination of the familiarity, accuracy, memorability, and concreteness.
D. Describe the ground: how are the subject and analog alike? How are they different?
E. Evaluate the analogy: does the analogy work with the intended audience?

Analogies have repeatedly been found to be effective in learning all types of subject matter. For example, many complex control systems are included among the functions of the human body. Driving a car is a way in which the human body functions as a complex controller and a suite of sensors and actuators. The eyes are the sensors that detect the position of the car and the center of the road. A complex controller, the brain, compares the two positions and determines which actions must be performed to accomplish the desired result. The body implements the control action by turning the steering wheel. An experienced driver will anticipate all types of disturbances to the system, such as a rough section of pavement or a slow-moving vehicle ahead [24].

In the electric circuits course, we have presented many analogies to students. Some of the analogies are well known, such as Johannes Kepler's analogy between gravitational field and electric field [20]. Others are novel, or build on existing analogies in novel ways. In what follows, we focus only on the novel analogies, as these are contributions to the literature, rather than repeating analogies that have been reported. The analogies (both existing and novel) are used both when the topic is introduced and repeatedly during analysis examples, where applicable, to help remind students about the underlying theoretical reasoning applied during the analysis.

The first example is explained in detail while the rest of the examples are presented in a concise manner for two purposes; one is to leave them as exercises for the reader and the other is to keep the paper limited in length. We also note that we have either abbreviated or omitted the discussion of the limitations and imprecisions of each analogy for space considerations. It is imperative however to discuss the limitations of the analogy with students to avoid misconceptions [19, Chapter 1]. In the course, many of the analogies are presented with associated imagery.

\section{Example 1: Analogy of Discharging and Charging a Capacitor}

The four parts of an analogy, described above, are incorporated to explain this analogy:

1. The target, or subject: the information to be learned.

The concept of discharging and charging a capacitor in an RC circuit containing a switch.

2. The source, or analog: the familiar thing to which the new information is compared.

The concept of flushing and filling the tank of a toilet. 
3. The connector: the means by which the subject and analog are compared.

The connectors in this analogy are presented as:

- The cross-sectional area of the water tank is similar to capacitance ( $C$ in Farads).

- The water volume in the tank is similar to the charge ( $Q$ in Coulomb).

- The level of the water in the tank that is measured by the floating device is similar to the voltage across the capacitor $\left(v_{c}=Q / C\right.$ in volts).

- The pipe connecting the supplied water to the tank is similar to a resistor that is inversely proportional to the cross section of the pipe ( $R_{1}$ in Ohms).

- The pipe that discharges the water from the tank is similar to a resistor $\left(R_{2}\right.$ in Ohms, where $R_{2} \ll R_{1}$ because the cross sectional area of the discharge pipe is much larger than the cross sectional area of the water supplying pipe).

- The difference in elevation between the pipe connecting the supplied water at the top of the tank and the pipe that discharges the water at the bottom of the tank is similar to an independent DC voltage source. $\left(V_{D C}=V_{\max }\right.$ : the maximum level of water in the tank in volts).

- The toilet tank flush lever handle that flushes the water tank and then closes after being released is similar to a switch (closes at $t=0$ (time of starting the flush or discharging) and opens at $t=T$ (time of starting the filling or charging).

4. The ground: the description of the similarities and differences between the target subject and analog, given below.

The description of the process and concept can be presented in a first-order circuit as shown in Figure 1 . The tank starts at its maximum water capacity, $V_{\max }$. When the flush lever handle is pressed, the water is flushed very fast, which is similar to the switch in the circuit, when it closes, the voltage drops quickly to $V_{\max } \frac{R_{2}}{R_{1}+R_{2}} \approx 0$ due to the fact that the discharge time constant $\tau_{2}=$ $R_{2} C$ is very small. When the flush lever is released at time $T$ the tank is filled slowly with water to its maximum capacity. The same as when the switch in the circuit opens at time $T$. The reason that the tank fills much slower than emptying it is due to the fact that $\tau_{1}=R_{1} C$ is much larger than $\tau_{2}$. When the water reaches the maximum capacity of the tank, it is completely shut down. The same as when the voltage of the capacitor reaches $V_{\max }$ there is no current passing through $R_{1}$ and the capacitor acts as an open circuit.

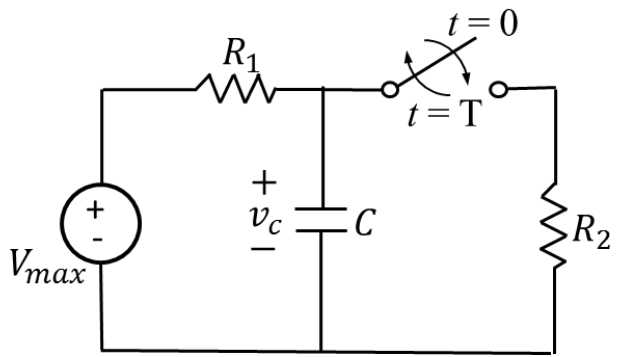

Figure 1 First-order circuit

A major limitation of this analogy is that a toilet lever does not need to be held down the entire duration of the draining process, so the switch model is imprecise. A second difference is that the fill valve of the toilet is gradually closed over a short duration as the float arm reaches its peak. This detail is missing from the model. 


\section{Example 2: Analogy of Power Supplied and Power absorbed}

For passive sign convention to determine an element if supplying or absorbing power, we use $P=-v i$, if the answer is a negative value then power is supplied. If the value is positive, then it is absorbed the same as if you give a friend $\$ 20$, you have supplied the money (negative for you!) and your friend has gained (or "absorbed") the $\$ 20$ (positive for him or her!). It is important to emphasize that for power, positive and negative denotes the sign of the value, whereas in the analog, positive and negative denotes feelings.

\section{Example 3: Analogy of Kirchhoff's Current Law}

The total number of cars entering an intersection is equal to the total number of cars leaving it as the total current entering a node is equal to the total current leaving it. By the analogy between conservation of mass and charge, the cars flowing through the intersection represent rate of mass, whereas current flowing through a node represents rate of charge. Hopefully the cars won't crash into the infrastructure as electrons collide within the molecular structure!

\section{Example 4: Analogy of Kirchoff's Voltage Law}

Altitude is a relative quantity and is associated with a potential energy due to the Earth's gravitational field. Similarly, voltage is associated with a potential energy due to an underlying electric field. Hence, altitude is analogous to voltage. Consider taking a hike up a mountain going on one path and then returning from the summit along another path. Suppose the return trail is along the other side of the mountain, and the return path eventually takes us back to our starting location. This is the same concept as a loop in a circuit. The altitude change from start to finish is of course 0 since we ended where we started. The same is true for the voltage around a loop! We call this a KVL hike in the circuit. In this analogy, wires are flat sections of terrain and different circuit elements result in different altitude changes. To deal with KVL equations that jump from one point in the circuit to another without directly traversing circuit elements (e.g., using a node voltage in a KVL involving ground), we talk about taking a helicopter between the nodes and then hiking the rest of the way.

\section{Example 5: Analogy of Series resistors and Voltage Division}

Kids on one team in tug of war pull side by side in a line to additively increase their resistance against the other team. Similarly, the current-resisting effects of series resistors combine to additively increase the overall resistance. As voltage is analogous to force, each teammate's force additively contributes to the overall force of the team. Hence, the ratio of the teammate's force to the team's force is equal to the ratio of the teammate's resistance against the other team to the total resistance. This equation is the voltage division equation for series resistors. It is helpful to include imagery of kids playing tug of war when introducing this analogy. It is also important to emphasize that although the forces in the tug of war game must be along a line for the analogy to hold, series resistors may take on random orientations as long as they are connected in series. It is helpful to show different orientations of series-connected resistors to help avoid misconceptions from being formed by this imprecision in the analogy. 


\section{Example 6: Analogy of Parallel Resistors}

Combining parallel resistors is like combining people working in parallel on a job. For example, suppose Mary takes 2 hours to paint a room and John takes 4 hours to paint it. How long would it take if they paint working in parallel? Clearly, it should take less than 2 hours, which is the least amount of time among the people working alone. It turns out that the product-over-the-sum rule works since there are exactly 2 people working in parallel. Hence, it takes $\frac{(2)(4)}{2+4}=\frac{8}{6}$ or 1.33 hours to complete the job (i.e., paint the room). Note that we should use the amount of time to complete the job in the calculations.

\section{Example 7: Analogy of Parallel Conductance}

From the parallel resistor analogy we see that the time required to complete a job for people working in parallel is calculated in the same way as parallel resistance when the resistance is matched to the amount of time each person requires to complete the job. The reciprocal of time per job is work rate (i.e., amount of a job done per unit time); hence we can relate conductance to work rate. For example, suppose Mary and John are cashiers at a grocery store and Mary can check out 15 customers per hour while John can check out 10 customers per hour (their work rates). Clearly, working in parallel, together, Mary and John can check out $15+10=25$ customers per hour.

\section{Example 8: Analogy of Circuit's Ground}

Building on the altitude analogy for voltage, altitude at each point on Earth is defined with respect to sea level. Similarly, each node voltage in a circuit is defined with respect to the ground node. The altitude of land at sea level is $0 \mathrm{~m}$. The voltage of the ground node is $0 \mathrm{~V}$. It is important to emphasize how voltages in a circuit may be below ground potential (i.e., negative). This can be incorporated into the analogy by talking about basements or underground shelters, but this limitation should be emphasized.

\section{Example 9: Analogy of Supermesh}

When taking a KVL hike, we avoid current sources just like we would avoid hiking up (or down) a waterfall! Instead, we must find another path that avoids hiking the waterfall. A supermesh is just an alternative loop that visits much of the same scenery (i.e., circuit elements) but avoids the current source. The emphasis for this analogy is to avoid current sources in loop analysis. It is helpful to invoke an image of a massive waterfall to emphasize the need to avoid it!

\section{Example 10: Analogy of Capacitor Construction and Limitations}

Consider a tank holding a compressible gas, such as a pressurized $\mathrm{CO} 2$ tank. The geometry of the tank determines how much compressed gas it can hold, as well as the material properties of the tank. Think of the material properties of the tank walls as similar to the permittivity of the dielectric. Changing the dielectric in the design of the capacitor would be like changing the material of the tank walls. The pressure in the tank is like the voltage across the capacitor. We may increase the pressure to increase the amount of gas (charge) that can be held in the tank, but 
there will be a critical pressure beyond which the tank will rupture (or explode). Similarly, there is a critical voltage, called the breakdown voltage of the capacitor, beyond which the capacitor will be damaged (or explode). Be careful never to exceed the breakdown voltage of the capacitor. An image of a propane tank or other gas holding tank is helpful when introducing this analogy.

\section{Example 11: Analogy of Inductor}

Inductors oppose changes in the current flowing through them because it requires a transfer of energy to change the magnetic field energy stored in the inductor. This is similar to how a wrecking ball transfers its kinetic energy associated with its velocity to a wall that it strikes (it is the force applied by the wrecking ball that destroys the wall; i.e., the energy transfer). Thus, we can relate the voltage across the inductor to the force applied to the wall by the moving wrecking ball, and we can relate the current through the inductor to the velocity of the wrecking ball. Just as a large wrecking ball creates a large force when it strikes a wall, an inductor with a large inductance induces a large voltage across it when its current is attempted to be changed quickly. In fact, when dealing with inductances in electronic design, it is important to protect other components in the circuit from this wrecking ball effect (called inductive kickback) that occurs when the current through the inductor is changed quickly through switching action. A wrecking ball image, possibly with a brief video, is helpful when introducing this analogy.

\section{Example 12: Analogy of RL Energy Dissipation and RL Time Constant}

Energized inductors are like masses in motion. Resistors model energy dissipation similar to friction in mechanical systems. Imagine a large big rig (eighteen-wheeled truck) traveling at moderate velocity and then trying to stop quickly. The larger the truck (mass), the longer it will take to stop (larger time constant). Also, weak brakes or poor road conditions (less friction in either case) will cause the truck to require more time to stop (larger time constant). An energized RL circuit is similar. The larger the inductance (like mass), the longer it will take to de-energize the inductor. Similarly, the smaller the equivalent resistance seen by the inductor (like the friction), the longer it will take to de-energize. Hence the time constant of the RL circuit is directly proportional to inductance and inversely proportional to equivalent resistance. Notice that it is the friction experienced by the truck that is important in the stopping process, just as it is the equivalent resistance seen by the inductor that is important in the RL energy dissipation process.

\section{Reflections and Conclusions}

In this paper we have described a set of classroom activities and assignments for an electric circuits course that target curiosity, creating value, and connections, i.e., elements of the entrepreneurial mindset. We have also outlined a dozen novel circuit analogies that have been popular with students. To demonstrate the popularity among students, we provide some student comments.

The standard end-of-course evaluation survey provided by the university to students includes three open-ended question prompts to allow students the opportunity to provide detailed 
feedback about the course. The first question prompt is "What were the strengths of the course? What did you enjoy most about the class? What teaching methodologies did you find most effective? Please provide specific examples where possible." Many of the student responses to this question prompt support the use of analogies in the course. Some examples taken from student feedback include:

- Very well structured and thought out. Though there was a load of material to cover it was all conveyed in a reasonable and effective manner. Analogies also helped in understanding all the material well.

- $\quad[\mathrm{T}]$ he analogies and homework were the best teaching methods.

- [The instructor] did a good job of relating information to students in real world examples and analogies that we can understand and comprehend.

- The analogies were very good at helping relate the concepts to prior knowledge.

- It was very helpful to have complex ideas explained with analogies.

- I really liked that [the instructor] would give us analogies to help us understand harder topics with topics we already understood.

The other two question prompts focus on weaknesses of the course and how the course could be improved. Importantly, there were no comments about the analogies under these question prompts. Also, there were no negative comments about the curiosity or creating value related assignments or activities.

From an assessment viewpoint, the average of the analogy reflection was a high B. Students improved their question formulation ability across the three question submission assignments by approximately a full letter grade $(10 \%)$, on average, according to the rubric. The assessment data for the design projects was strong for the analysis, simulation, and test plans, but poor for the value propositions. This suggests more emphasis should be placed on developing value propositions grounded with credible and convincing data and facts. Future work includes development of value proposition exercises specific to electric circuits topics (e.g., develop a value proposition for the implementation of circuit breakers over plug fuses for residential wiring) and the development of programmatic assessment of the entrepreneurially minded learning outcomes of the KEEN framework.

\section{References}

[1] E. M. Eisenstein, "Engineering and entrepreneurship: Creating lasting value from engineering," in IEEE Transforming Engineering Education: Creating Interdisciplinary Skills for Complex Global Environments, Dublin, 2010, pp. 1-15.

[2] "KEEN - Home," KEEN - Engineering Unleashed. [Online]. Available: https://engineeringunleashed.com/. [Accessed: 30-Jan-2019].

[3] D. Rae and D. E. Melton, "Developing an entrepreneurial mindset in US engineering education: an international view of the KEEN project," The Journal of Engineering Entrepreneurship, 7(3), 2017. 
[4] T. G. Reio Jr, and A. Wiswell, "Field investigation of the relationship among adult curiosity, workplace learning, and job performance," Human Resource Development Quarterly, 11(1), pp. 5-30, 2000.

[5] T. J. Kriewall and K. Makemson, "Instilling the entrepreneurial mindset into engineering undergraduates," The Journal of Engineering Entrepreneurship, 1(1), pp. 5-19, July 2010.

[6] J. Wheadon and N. Duval-Couetil, "Elements of entrepreneurially minded learning: KEEN white paper," The Journal of Engineering Entrepreneurship, 7(3), pp. 17-25, 2016.

[7] A. Gerhart and D. E. Melton, "Entrepreneurially minded learning: Incorporating stakeholders, discovery, opportunity identification, and value creation into problem-based learning modules with examples and assessment specific to fluid mechanics," The Journal of Engineering Entrepreneurship, 8(1), pp. 44-62, 2017.

[8] T. J. van Schijndel, B. Jansen, and M. Raijmakers. "Do individual differences in children's curiosity relate to their inquiry-based learning?," International Journal of Science Education, 40(9), pp. 996-1015, 2018.

[9] R. Duit, "On the role of analogies and metaphors in learning sciences," Science Education, 75(6), pp. 649-672, 1991.

[10] D. E. Brown, "Refocusing core intuitions: A concretizing role for analogy in conceptual change," Journal of Research in Scientific Teaching, 30(10), pp. 1273-1290, 1993.

[11] B. Leavy, "Collaborative innovation as the new imperative - design thinking, value cocreation and the power of "pull"," Strategy \& Leadership, 40(2), pp. 25-34, 2012.

[12] C. L. Dym, A. M. Agogino, O. Eris, D. D. Frey, and L. J. Leifer, "Engineering design thinking, teaching, and learning," Journal of Engineering Education, 94(1), pp. 103-120, 2005.

[13] M. J. de Almeida, A. Salvador, and M. M. Costa, "Analogy for Drude's free electron model to promote students' understanding of electric circuits in lower secondary school," Physical Review Special Topics-Physics Education Research, 10(2), 020118, 2014.

[14] D. Black and J. Solomon, "Can pupils use taught analogies for electric current?," School Science Review, 69, pp. 249-254, 1987.

[15] S. M. Glynn and T. Takahashi, "Learning from analogy-enhanced science text," Journal of Research in Science Teaching, 35(10), pp. 1129-1149, 1998.

[16] D. R. Gentner and D. Gentner, "Flowing waters or teeming crowds: Mental models of electricity," Mental models, pp. 99-129, 1983.

[17] S. M. Glynn, "Explaining science concepts: A teaching-with-analogies model," The Psychology of Learning Science, pp. 219-240, 1991.

[18] R. J. Spiro, P. J. Feltovich, R. L. Coulson, and D. K. Anderson, "Multiple analogies for complex concepts: Antidotes for analogy-induced misconception in advanced knowledge acquisition," In S. Vosniadou \& A. Ortony (Eds.), Similarity and analogical reasoning. Cambridge: Cambridge University Press, pp. 498-531, 1989.

[19] D. L. Schwartz, J. M. Tsang, and K. P. Blair. The ABCs of How We Learn: 26 Scientifically Proven Approaches, How They Work, and When to Use Them. W. W. Norton \& Company, Inc., New York, NY, 2016.

[20] D. Gentner, S. Brem, R. W. Ferguson, A. B. Markman, B. B. Levidow, P. Wolff, and K. D. Forbus, "Analogical reasoning and conceptual change," Journal of the Learning Sciences, 6(1), pp. 3-40, 1997. 
[21] J. K. Estell and S. Howe, "Development and use of a client interaction rubric for formative assessment," In Proceedings of the 2017 ASEE Annual Conference \& Exposition, Columbus, OH, June 2017.

[22] F. Hassan, H. LeBlanc, and K. Al-Olimat, "Inculcating an entrepreneurial mindset in engineering education: Project approach," In IEEE Frontiers in Education Conference, pp. 121-126, 2013.

[23] C. Kearny, T. Newby, and D. Stepich, "Building bridges: Creating instructional analogies," presentation at the Annual Convention of the National Society for Performance and Instruction, Atlanta, GA, March 1995.

[24] S. Kamalasadan, K. S. Al-Olimat, Fuzzy Logic Control Systems, Invited Book Chapter, Encyclopedia of Computer science and Engineering, edited by Benjamin Wah. John Wiley \& Sons, Hoboken, NJ, ISBN: 978-0-471-38393-2, Vol 2, pp. 1344-1355, March 2009. 